Revista de MatemáticA: TeORÍA y APliCACiOnes 2018 25(2) : 319-345

CIMPA - UCR ISSN: 1409-2433 (PRINT), 2215-3373 (ONLINE)

DoI: https://doi.org/10.15517/rmta.v25i2.33617

\title{
TEORÍA CUASILINEAL DE KATO
}

\section{QUASILINEAR THEORY OF KATO}

\author{
CÉSAR LOZA ROJAS*
}

Received: 14/Jun/2017; Revised: 28/Apr/2018;

Accepted: 27/Apr/2018

Revista de Matemática: Teoría y Aplicaciones is licensed under a Creative Commons Reconocimiento-NoComercial-Compartirigual 4.0 International License.

* Departmento de Matemática, Universidad Nacional San Luis Gonzaga, Ica, Perú. E-Mail: lozacr@gmail.com 


\title{
Resumen
}

En el presente artículo analizaremos el problema de Cauchy local asociado a la ecuación de Korteweg-De Vries (KdV) en $H^{s}$ con $s>3 / 2$. El objetivo de este trabajo, consiste en establecer la buena formulación local del problema cuando $u_{0} \in H^{s}, s>3 / 2$, para ello aplicaremos la teoría cuasi-lineal de Kato, el cual consta de (06) hipótesis, en el caso lineal y (08) hipótesis en el caso no-lineal. En la solución del problema de Cauchy para la ecuación de evolución cuasi-lineal, nos basaremos en el teorema del punto fijo de Banach.

Palabras clave: teorema de existencia local y unicidad; existencia de soluciones generalizadas; aplicaciones de EDP en áreas distintas de la física.

\begin{abstract}
In the present paper we will analyze the local Cauchy problem associated with the Korteweg-De Vries (KdV) equation in $H^{s}$ with $s>3 / 2$. The objective of this work is to establish the good local formulation of the problem when $u_{0} \in H^{s}, s>3 / 2$, for this we apply the quasi-linear theory of Kato, which consists of (06) hypotheses, in the linear case and (08) hypotheses in the non-linear case. In the solution of Cauchy's problem for the quasi-linear equation of evolution, we will rely on Banach's fixed-point theorem.
\end{abstract}

Keywords: local existence and uniqueness theorems; existence of generalized solutions; applications of PDE in areas other than physics.

Mathematics Subject Classification: 35A07, 35D05, 35Q80. 


\section{Introducción}

Basándonos en nuestra experiencia con ecuaciones diferenciales lineales y pensando en los resultados que vamos a describir en este trabajo, formulamos las preguntas fundamentales que se plantean en el estudio del problema de Cauchy

$$
\left\{\begin{array}{l}
\partial_{t} u(t)=F(t, u(t)) \in X \\
u(0)=\varphi \in Y
\end{array}\right.
$$

en donde $X$ e $Y$ son espacios de Banach, $\left.T_{0} \in\right] 0, \infty\left[\right.$ y $F:\left[0, T_{0}\right] \times Y \rightarrow X$ es una función:

P-1 Existen $\left.T \in] 0, T_{0}\right]$ y una función $u \in C([0, T], Y)$ de tal manera que $u(0)=\varphi$ y la ecuación diferencial se cumple en el sentido de que

$$
\lim _{h \rightarrow 0}\left\|\frac{u(t+h)-u(t)}{h}-F(t, u(t))\right\|_{X}=0
$$

en donde las derivadas en $t=0$ y $t=T$ se calculan a la derecha y a la izquierda, respectivamente.

P-2 El problema (1), tiene al menos una solución en $C([0, T], Y)$.

P-3 La aplicación $\varphi \mapsto u$ es continua. Con mayor precisión, si $u \in C\left(\left[0, T^{*}\right], Y\right)$ es la solución del problema con valor inicial $\varphi \in Y$, sean $\varphi_{n} \in Y, n \in \mathbb{N}$ tales que $\varphi_{n} \stackrel{Y}{\longrightarrow} \varphi$ y $u_{n} \in C\left(\left[0, T_{n}\right], Y\right)$ las correspondientes soluciones. Sea $T \in] 0, T^{*}$ [, entonces las soluciones $u_{n}$ se pueden extender al intervalo a $[0, T]$ para todos los $n$ suficientemente grandes y

$$
\lim _{n \rightarrow+\infty} \sup _{[0, T]}\left\|u_{n}(t)-u(t)\right\|_{Y}=0 .
$$

El estudio de las preguntas anteriores será denominado el problema básico para (1). En caso de que las respuestas a las preguntas anteriores sean afirmativas el problema de Cauchy (1) será llamado bien formulado localmente. Si cualquiera de estas condiciones no se cumple, decimos que el problema está mal formulado.

Rev.Mate.Teor.Aplic. (ISSN print: 1409-2433; online: 2215-3373) Vol. 25(2): 319-345, Jul-Dec 2018 
Si (1) está definida en $\left[0,+\infty\left[\right.\right.$ y $\left(\mathrm{P}_{1}\right),\left(\mathrm{P}_{2}\right)$ y $\left(\mathrm{P}_{3}\right)$ son válidas en $[0, T]$ para todo $T>0$ diremos que (1) es bien formulado globalmente.

\section{Problema básico para un problema de Cauchy}

Sea la ecuación de evolución, ecuación de Korteweg-De Vries (Kdv)

$$
\left\{\begin{array}{l}
\partial_{t} u(x, t)+\partial_{x}^{3} u(x, t)+u^{p}(x, t) \partial_{x} u(x, t)=0 \\
u(x, 0)=u_{0},
\end{array}\right.
$$

donde $u$ es una función con valores reales para $x \in \mathbb{R}$ y $t \geq 0$, y $p$ es un entero positivo.

El objetivo de este artículo, es establecer la buena formulación local del problema cuando $u_{0} \in H^{s}, s>3 / 2$. Para ello aplicaremos la Teoría cuasi-lineal de Kato, la cual pasamos a exponer.

Consideremos el problema de Cauchy para la ecuación de evolución cuasilineal

$$
\left\{\begin{array}{l}
\partial_{t} u+A(t, u) u=f(t, u) \\
u(0)=\varphi
\end{array}\right.
$$

en un espacio de Banach $X$. Llamaremos a $A(t, u) u$ parte cuasi-lineal y $f(t, u)$ la parte semi-lineal de la ecuación dada.

A grandes rasgos, queremos resolver (5) de la siguiente manera. Para ciertas funciones $t \rightarrow v(t)$, consideramos la ecuación lineal

$$
\left\{\begin{array}{l}
\partial_{t} u+A(t, v(t)) u=f(t, v(t)) \\
u(0)=\varphi
\end{array}\right.
$$

Si (6) tiene una solución $u=u(t)$, hemos definido una aplicación $v \mapsto u=\Phi v$. Luego buscamos un punto fijo de $\Phi$, que será una solución de (5). Para demostrar que $\Phi$ tiene un punto fijo se utiliza el teorema del punto fijo de Banach. 
Con este fin consideremos las siguientes condiciones referidas al problema de Cauchy (5):

(X) Sean $X$ e $Y$ dos espacios de Banach reflexivos tales que $Y$ está contenido densamente y continuamente en $X$. Además, existe un isomorfismo $S: Y \rightarrow X$ y la norma de $Y$ es escogida de forma que $S$ sea una isometría.

A-1 $A(\cdot, \cdot)$ es un operador definido en $\left[0, T_{0}\right] \times W$ con valores en $\mathcal{G}(X: 1, \omega)$, siendo $W$ una bola abierta en $Y$ y $\omega$ un número real. Es decir, para cada $(t, y) \in\left[0, T_{0}\right] \times W,-A(t, y)$ es el generador de un semigrupo fuertemente continuo en $X$ tal que

$$
\left\|e^{-s A(t, y)}\right\|_{\mathcal{L}(X)} \leq e^{\omega s}, \quad s \geq 0, t \in\left[0, T_{0}\right], y \in W
$$

A-2 Para cada $(t, y) \in\left[0, T_{0}\right] \times W$, tenemos

$$
S A(t, y) S^{-1}=A(t, y)+B(t, y),
$$

donde $B(t, y) \in \mathcal{L}(X)$ y $\|B(t, y)\|_{\mathcal{L}(X)} \leq \lambda_{B}$ con $\lambda_{B}>0$ una constante. Además, existe $\mu_{B}>0$ tal que

$$
\left\|B\left(t, y_{1}\right)-B\left(t, y_{2}\right)\right\|_{\mathcal{L}(X)} \leq \mu_{B}\left\|y_{1}-y_{2}\right\|_{Y},
$$

para todo $t \in\left[0, T_{0}\right]$ y $y_{1}, y_{2} \in W$.

A-3 Para cada $(t, y) \in\left[0, T_{0}\right] \times W$ tenemos que $A(t, y) \in \mathcal{L}(Y, X)$, en el sentido que $Y \subseteq \mathcal{D}(A(t, y))$ y $\left.A(t, y)\right|_{Y} \in \mathcal{L}(Y, X)$. Además, para cada $y \in W$ la aplicación $t \in\left[0, T_{0}\right] \mapsto A(t, y)$ es fuertemente continua.

A-4 Para cada $t \in\left[0, T_{0}\right]$ la aplicación $y \in W \mapsto A(t, y)$ es Lipschitz continua en $\mathcal{L}(Y, X)$; es decir, existe $\mu_{A}>0$ tal que

$$
\left\|A\left(t, y_{1}\right)-A\left(t, y_{2}\right)\right\|_{\mathcal{L}(X)} \leq \mu_{A}\left\|y_{1}-y_{2}\right\|_{Y}
$$

para todo $t \in\left[0, T_{0}\right]$ y $y_{1}, y_{2} \in W$. 
A-5 Existe $\mu_{3}>0$ tal que

$$
\|Q(t, y)-Q(t, z)\|_{\mathcal{L}(X)} \leq \mu_{3}\|y-z\|_{Y} .
$$

f-1 $f:[0, T] \times W \rightarrow Y$ es acotada

$$
\|f(t, y)\|_{Y} \leq \lambda_{3}, \quad t \in[0, T], \quad y \in W .
$$

Para cada $y \in W, t \longmapsto f(t, y)$ es continua de $[0, T]$ en $\mathrm{X}$ mientras que para cada $t \in[0, T]$ la aplicación $y \in W \longmapsto f(t, y)$ es Lipschitz en X esto es,

$$
\|f(t, y)-f(t, z)\|_{X} \leq \mu_{2}\|y-z\|_{X},
$$

donde $\mu_{2} \geq 0$ una constante.

f-2 Existe $\mu_{4}>0$ tal que

$$
\|f(t, y)-f(t, z)\|_{Y} \leq \mu_{4}\|y-z\|_{Y},
$$

para todo $t \in[0, T], y, z \in W$.

Ahora podemos enunciar el teorema de buena formulación local de Kato.

\section{Teorema 2.1}

1. (Existencia y unicidad). Si las condiciones (X), (A1)-(A4) y (f1) y (f2) se cumplen y $\varphi \in W$, el problema de Cauchy (5) tiene solución única

$$
u \in C([0, T]: W) \cap C^{1}\left(\left[0, T^{\prime}\right]: X\right),
$$

para algún $T^{\prime}>0, T^{\prime} \leq T$.

2. (Dependencia continua respecto al valor inicial). Además del problema de Cauchy (5) consideremos la sucesión de problemas de Cauchy

$$
\left\{\begin{array}{l}
\partial_{t}^{n} u+A^{n}\left(t, u^{n}\right) u^{n}=f^{n}\left(t, u^{n}\right), 0 \leq t \leq T \\
u^{n}(0)=\varphi^{n}
\end{array}\right.
$$

$\operatorname{con} n=1,2, \ldots$ 
Supongamos que:

(a) Para las funciones $A^{n}$ y $f^{n}$ se cumplen las condiciones de (A1A5), (f1) y (f2) uniformemente en $n$, con los mismos espacios $X e$ $Y$, el mismo isomorfismo $S$ y la misma bola $W$, y para cada $(t, y) \in$ $[0, T] \times W$

$$
\begin{aligned}
& A^{n}(t, y) \rightarrow A(t, y) \quad \text { fuertemente en } \mathcal{L}(Y, X), \\
& B^{n}(t, y) \rightarrow B(t, y) \quad \text { fuertemente en } \mathcal{L}(X), \\
& f^{n}(t, y) \rightarrow f(t, y) \quad \text { en } Y, \text { cuando } n \rightarrow \infty .
\end{aligned}
$$

(b) Si $\varphi, \varphi_{n} \in W$ y $\varphi_{n} \rightarrow \varphi$ en la norma Y cuando $n \rightarrow+\infty$, existe un $T^{\prime \prime}>0, T^{\prime \prime}<T$, soluciones únicas

$$
u^{n} \in C\left(\left[0, T^{\prime \prime}\right]: W\right) \cap C^{1}\left(\left[0, T^{\prime \prime}\right]: X\right)
$$

para (9), $n=1,2, \ldots$ y una solución única u para (5) en la misma clase, tales que

$$
u^{n}(t) \rightarrow u(t) \text { en } Y \text {, uniformemente en } t \in\left[0, T^{\prime \prime}\right]
$$

\section{Observaciones}

1. Condición (A4) es satisfecha trivialmente si $y_{0}=0$.

2. En muchos casos $\mathrm{A}(t, y)$ se define para todo $y \in Y$, de modo que la bola $\mathrm{W}$ puede ser elegida con centro en cero y radio arbitrario, a pesar de las constantes $\beta, \lambda_{1}, \mu_{1}, \cdots$ dependerá del radio de la bola.

3. Una condición suficiente para (7) es

$$
\left\|[S A(t, y)-A(t, y) S] S^{-1} w\right\|_{X}<\lambda_{1}\|w\|_{X}
$$

para todos los w en un núcleo de $A(t, y)$ (que puede depender de $t$ e $y$ ). 
4. El enunciado de la condición (A3) fue:

Para cada $(t, y) \in\left[0, T_{0}\right] \times W$ tenemos que $A(t, y) \in \mathcal{L}(Y, X)$, en el sentido que $Y \subseteq \mathcal{D}(A(t, y))$ y $\left.A(t, y)\right|_{Y} \in \mathcal{L}(Y, X)$. Además, para cada $y \in W$ la aplicación $t \in\left[0, T_{0}\right] \mapsto A(t, y)$ es continua en la norma de $\mathcal{L}(Y, X)$.

\section{El problema de Cauchy lineal}

En esta sección estudiaremos el problema lineal determinado por (4)

$$
\left\{\begin{array}{l}
\partial_{t} u(x, t)+\partial_{x}^{3} u(x, t)=0 \\
u(x, 0)=u_{0}
\end{array}\right.
$$

Para esto definimos el operador $B$ por

$$
\left\{\begin{array}{l}
\mathcal{D}(B)=H^{s+3}, s \geq 0 \\
B u=-\partial_{x}^{3} u, u \in H^{s} .
\end{array}\right.
$$

Así el problema lineal (10) puede escribirse en la forma

$$
\left\{\begin{array}{l}
\partial_{t} u-B u=0, x \in \mathbb{R}, t>0 \\
u(0)=u_{0}
\end{array}\right.
$$

Proposición 3.1 Para todo $s \geq 0, B: H^{s+3} \subseteq H^{s} \rightarrow H^{s}$ es un operador lineal con dominio denso y anti-adjunto. En particular, $B$ y $-B$ son operadores m-disipativos en $H^{s}$.

Demostración : La linealidad del operador B es inmediata. Además, si $u \in H^{s+3}$ tenemos

$$
\|B u\|_{H^{s}} \leq\left\|J^{s+3} u\right\|_{L^{2}}=\|u\|_{H^{s+3}} .
$$

Entonces $B u \in H^{s}$ cualquiera sea $u \in H^{s+3}$. 
Veamos que B es antiadjunto. En efecto, si $u \in H^{s+3} y v \in H^{s}$ tenemos

$$
\langle B u, v\rangle_{H^{s}}=\left\langle-\partial_{x}^{3} u, v\right\rangle_{H^{s}}=(-1)^{3}\left\langle u,-\partial_{x}^{3} v\right\rangle_{H^{s}}=-\langle u, B v\rangle_{H^{s}},
$$

donde se ha usado la propiedad $\left\langle\partial_{x}^{k} u, v\right\rangle_{H^{s}}=(-1)^{k}\left\langle u, \partial_{x}^{k} v\right\rangle_{H^{s}}$ válida para todo $u, v \in H^{s}$. Para el resto de la prueba usamos la proposición 5.5 de [9].

De este modo obtenemos el resultado principal del caso lineal.

Teorema 3.1 El operador lineal $B$ genera un semigrupo de contracciones $\left\{e^{-t \partial_{x}^{3}}\right\}_{t \geq 0}$, en $H^{s}$ para cualquier $s \in \mathbb{R}$, tal que

$$
\widehat{e^{-t \partial_{x}^{3}} u}(\xi)=e^{i t \xi^{3}} \widehat{u}(\xi)
$$

para todo $u \in H^{s}$. Además, $\left\{e^{-t \partial_{x}^{3}}\right\}_{t \geq 0}$, puede ser extendido a un grupo de operadores fuertemente continuos en $H^{s} y$, cualquiera sea $u_{0} \in H^{s}$ la función

$$
e^{-(\cdot) \partial_{x}^{3}} u:\left[0,+\infty\left[\rightarrow H^{s}\right.\right.
$$

es la única solución del problema (12).

Demostración : La primera afirmación es una simple consecuencia de la proposición 3.1, y el Teorema de Lumer-Phillips [10, Teorema 5.3]. Para obtener (13) es suficiente tomar la transformada de Fourier en la variable espacial y usar la proposición 6.1 de [10].

Antes de concluir la sección notemos que en este caso no es posible resolver el problema (4) de manera tradicional, es decir, reducirlo a una ecuación integral y aplicar el teorema del punto fijo de Banach. En efecto, es fácil verificar que (4) es, al menos formalmente, equivalente a

$$
u(t)=e^{-t \partial_{x}^{3}} \varphi-\int_{0}^{t} e^{-(t-\tau) \partial_{x}^{3}} F(u(\tau)) d \tau,
$$

en donde $\left\{e^{-t \partial_{x}^{3}}\right\}_{t \geq 0}$, es el semigrupo de contracciones en $H^{s}, s \in \mathbb{R}$, generado por el operador matricial $B$ y $F(u)=u^{p} \partial_{x} u$.

Rev.Mate.Teor.Aplic. (ISSN print: 1409-2433; online: 2215-3373) Vol. 25(2): 319-345, Jul-Dec 2018 
Ahora, si $u \in C\left([0, T], H^{s}\right)$ entonces $F(u) \in C\left([0, T], H^{s-1}\right)$ y $e^{-(t-\tau) \partial_{x}^{3}}$ aplica $H^{s-1}$ en sí mismo y no en $H^{s}$. En consecuencia, la aplicación

$$
(\Psi u)(t)=e^{-t \partial_{x}^{3}} \varphi-\int_{0}^{t} e^{-(t-\tau) \partial_{x}^{3}} F(u(\tau)) d \tau,
$$

no transforma $C\left([0, T], H^{s}\right)$ en sí mismo de modo que el teorema del punto fijo de Banach no puede ser aplicado. En la próxima sección, mostraremos como la teoría de Kato permite restablecer el método tradicional.

\section{Buena formulación local en $H^{s}$ para $s>3 / 2$}

En teorema (5.3) si $n=1$ y $k=1$, tenemos $s>3 / 2$.

Para estudiar la buena formulación local de (4) usaremos la teoría de Kato, teorema (2.1). Para esto hacemos el cambio de variable

$$
u(t)=e^{-t \partial_{x}^{3}} v(t)
$$

De este modo

$$
\begin{aligned}
& \partial_{t} u(t)=-e^{-t \partial_{x}^{3}} \partial_{x}^{3} v(t)+e^{-t \partial_{x}^{3}} \partial_{t} v(t), \\
& \partial_{x} u(t)=e^{-t \partial_{x}^{3}} \partial_{x} v(t)=\partial_{x} e^{-t \partial_{x}^{3}} v(t), \\
& \partial_{x}^{3} u(t)=e^{-t \partial_{x}^{3}} \partial_{x}^{3} v(t)
\end{aligned}
$$

entonces

$$
\begin{aligned}
& \partial_{t} u(t)+\partial_{x}^{3} u(t)+u^{p}(t) \partial_{x} u(t) \\
= & {\left[-e^{-t \partial_{x}^{3}} \partial_{x}^{3} v(t)+e^{-t \partial_{x}^{3}} \partial_{t} v(t)\right]+e^{-t \partial_{x}^{3}} \partial_{x}^{3} v(t)+\left(e^{-t \partial_{x}^{3}} v(t)\right)^{p} \partial_{x} e^{-t \partial_{x}^{3}} v(t) } \\
= & e^{-t \partial_{x}^{3}} \partial_{t} v(t)+\left(e^{-t \partial_{x}^{3}} v(t)\right)^{p} \partial_{x} e^{-t \partial_{x}^{3}} v(t) \\
= & e^{-t \partial_{x}^{3}}\left[\partial_{t} v(t)+e^{t \partial_{x}^{3}}\left(e^{-t \partial_{x}^{3}} v(t)\right)^{p} \partial_{x} e^{-t \partial_{x}^{3}} v(t)\right]
\end{aligned}
$$

y

$$
u_{0}=u(0)=e^{-(0) \partial_{x}^{3}} v(0)=I v(0)=v(0)
$$


Por tanto, obtenemos el problema

$$
\left\{\begin{array}{l}
\partial_{t} v(t)+A(t, v(t)) v(t)=0, t \geq 0 \\
v(0)=u_{0}
\end{array}\right.
$$

en donde $A$ es un operador lineal que depende de $(t, y) \in\left[0,+\infty\left[\times H^{s}\right.\right.$ definido por

$$
A(t, y)=e^{t \partial_{x}^{3}} M_{t} y \partial_{x} e^{-t \partial_{x}^{3}}
$$

$\operatorname{con} M_{t} y=\left(e^{-t \partial_{x}^{3}} y\right)^{p}$.

A continuación verificaremos las hipótesis del teorema (2.1) para el problema de valor inicial (14) en el espacio $H^{s}$ con $s>3 / 2$.

\subsection{Hipótesis $(\mathrm{X})$}

Sean $s>3 / 2$,

$$
X=L^{2} \quad \text { y } \quad Y=H^{s}
$$

Sabemos que $Y$ está contenido en $X$ densamente y continuamente. Definimos sobre $Y$ el operador $S$ por

$$
S u=J^{s} u, \quad u \in Y,
$$

en donde $J^{s}=\left(1-\partial_{x}^{2}\right)^{s / 2}$ es el potencial de Bessel de orden $s$, así para $u \in Y$

$$
\widehat{S u}(\xi)=\left(1+\xi^{2}\right)^{s / 2} \widehat{u}(\xi) .
$$

Proposición 4.1 $S \in \mathcal{L}(Y, X)$ es un isomorfismo isométrico.

Demostración : Es obvio que S es un operador lineal y como

$$
\|S u\|_{X}^{2}=\left\|\widehat{J^{s} u}\right\|_{L^{2}}^{2}=\int_{\mathbb{R}}\left(1+\xi^{2}\right)^{s / 2}|\widehat{u}(\xi)|^{2} d \xi=\|u\|_{H^{s}}^{2}=\|u\|_{Y}^{2},
$$

entonces $S: Y \rightarrow X$ es un operador lineal isométrico, así existe $S^{-1}: \mathcal{R}(S) \rightarrow Y$ operador inverso de $S$. 
Además, dado $v \in L^{2}$ si definimos $u=J^{-s} v$ tenemos

$$
S u=J^{s}\left(J^{-s} v\right)=J^{0}(v)=v
$$

y

$$
\|u\|_{Y}=\left\|J^{-s} v\right\|_{H^{s}}=\left\|J^{s}\left(J^{-s} v\right)\right\|_{L^{2}}=\|v\|_{L^{2}}
$$

por lo tanto $u \in H^{s}$. En consecuencia $S$ es un operador lineal sobreyectivo.

Esto prueba la hipótesis $(X)$.

\subsection{Hipótesis (A1)}

Dado $u_{0} \in H^{s}$, sea $R>\left\|u_{0}\right\|_{s}$ un número real fijo y consideremos la bola

$$
W=B_{R}(0)=\left\{v \in H^{s}:\|v\|_{H^{s}}<R\right\}
$$

Antes de continuar con la verificación de la hipótesis (A1), observemos que $\partial_{x} e^{-t \partial_{x}^{3}} y^{p}$ es continua y acotada, pues $(\cdot)^{p} \in C^{\infty}(\mathbb{R}, \mathbb{R})$ y $y \in W$. En efecto, notemos que $\partial_{x} e^{-t \partial_{x}^{3}} y \in H^{s-1}$ ya que $y \in W \subset H^{s} \subset C_{\infty}^{1}$ pues $s>3 / 2$, y

$$
\begin{aligned}
\left\|\partial_{x} e^{-t \partial_{x}^{3}} y\right\|_{L^{\infty}} & \leq\left\|e^{-t \partial_{x}^{3}} \partial_{x} y\right\|_{L^{\infty}} \leq\left\|e^{-t \partial_{x}^{3}} \partial_{x} y\right\|_{H^{s-1}} \\
& \leq\left\|\partial_{x} y\right\|_{H^{s-1}} \leq\|y\|_{H^{s}} \leq R .
\end{aligned}
$$

Así

$$
\begin{aligned}
\left\|\partial_{x} M_{t} y\right\|_{L^{\infty}} & =\sup _{x \in \mathbb{R}}\left|\partial_{x} M_{t} y(x)\right|=\sup _{x \in \mathbb{R}} \mid \partial_{x} M_{t} y(x) \partial_{x} e^{-t \partial_{x}^{3} y(x) \mid} \\
& \leq\left\|\partial_{x}\left(M_{t} y\right)^{\frac{1}{p}}\right\|_{L^{\infty}} \sup _{x \in \mathbb{R}}\left|\partial_{x} M_{t} y(x)\right| \\
& \leq 2 \alpha R,
\end{aligned}
$$

en donde $\alpha=\sup _{x \in \mathbb{R}}\left|\partial_{x} M_{t} y(x)\right|<\infty$. 
Proposición 4.2 A es un operador definido en $[0,+\infty[\times W$ con valores en $\mathcal{G}(X: 1, w)$, siendo $w$ un número real. Es decir, para cada $(t, y) \in[0,+\infty[\times W,-A(t, y)$ es el generador de un semigrupo fuertemente continuo en $X$ tal que

$$
\left\|e^{-s A(t, y)}\right\|_{\mathcal{L}(X)} \leq e^{\omega s}, \quad s \geq 0, t \in[0,+\infty], y \in W .
$$

Demostración : Para todo $u \in C_{0}^{\infty}$ tenemos

$$
\begin{aligned}
\left\langle M_{t} y \partial_{x} u, u\right\rangle_{X} & =\left\langle M_{t} y \partial_{x} u, u\right\rangle_{L^{2}} \\
& =\frac{1}{2} \int_{\mathbb{R}} M_{t} y(x) \partial_{x} u^{2}(x) d x \\
& =-\frac{1}{2} \int_{\mathbb{R}} \partial_{x}\left(M_{t} y(x)\right) u^{2}(x) d x \\
& \geq-\frac{1}{2}\left\|\partial_{x} M_{t} y\right\|_{L^{\infty}} \int_{\mathbb{R}} u^{2}(x) d x \\
& =-\alpha R\|u\|_{L^{2}}=-\alpha R\|u\|_{X} .
\end{aligned}
$$

Por otro lado, dado que $\left\{e^{-t \partial_{x}^{3}}\right\}_{t \geq 0}$, es un grupo de operadores unitarios en $L^{2}$ tenemos

$$
\begin{aligned}
\langle A(t, y) u, u\rangle_{X} & =\langle A(t, y) u, u\rangle_{L^{2}} \\
& =\left\langle e^{t \partial_{x}^{3}} M_{t} y \partial_{x} e^{-t \partial_{x}^{3}} u, u\right\rangle_{L^{2}} \\
& =\left\langle M_{t} y \partial_{x} e^{-t \partial_{x}^{3}} u, e^{-t \partial_{x}^{3}} u\right\rangle_{L^{2}}
\end{aligned}
$$

En consecuencia,

$$
\langle A(t, y) u, u\rangle_{X}=\left\langle M_{t} y \partial_{x} e^{-t \partial_{x}^{3}} u, e^{-t \partial_{x}^{3}} u\right\rangle_{L^{2}} \geq-\alpha R\left\|e^{-t \partial_{x}^{3}} u\right\|_{X}=-\alpha R\|u\|_{X} .
$$

La demostración de está proposición se concluye por un argumento de densidad.

Esta proposición prueba la hipótesis (A1). 


\subsection{Hipótesis (A2)}

Notemos que si $u \in \mathcal{S}$ entonces $S u \in \mathcal{S}$,

$$
\partial_{x}^{k} S u=S \partial_{x}^{k} u \text { para } u \in \mathcal{S} \text { y } k \in \mathbb{N}
$$

y

$$
S e^{ \pm t \partial_{x}^{3}} u=e^{ \pm t \partial_{x}^{3}} S u
$$

Luego si $u \in \mathcal{S}$ tenemos por (15) y (16) que

$$
\begin{aligned}
{[S, A(t, y)] u } & =(S A(t, y)-A(t, y) S) u \\
& =\left(S e^{t \partial_{x}^{3}} M_{t} y \partial_{x} e^{-t \partial_{x}^{3}}-e^{t \partial_{x}^{3}} M_{t} y \partial_{x} e^{-t \partial_{x}^{3}} S\right) u \\
& =e^{t \partial_{x}^{3}} S M_{y} \partial_{x} e^{-t \partial_{x}^{3}} u-e^{t \partial_{x}^{3}} M_{t} y \partial_{x} S e^{-t \partial_{x}^{3}} u \\
& =e^{t \partial_{x}^{3}} S M_{y} \partial_{x} e^{-t \partial_{x}^{3}} u-e^{t \partial_{x}^{3}} M_{t} y S \partial_{x} e^{-t \partial_{x}^{3}} u \\
& =e^{t \partial_{x}^{3}}\left(S M_{y}-M_{t} y S\right) \partial_{x} e^{-t \partial_{x}^{3}} u \\
& =e^{t \partial_{x}^{3}}\left[S, M_{t} y\right] \partial_{x} e^{-t \partial_{x}^{3}} u
\end{aligned}
$$

En consecuencia

$$
[S, A(t, y)] S^{-1} u=e^{t \partial_{x}^{3}}\left[S, M_{t} y\right] \partial_{x} e^{-t \partial_{x}^{3}} S^{-1} u=e^{t \partial_{x}^{3}}\left[S, M_{t} y\right] \partial_{x} S^{-1} e^{-t \partial_{x}^{3}} u
$$

Probamos ahora el siguiente estimado.

Proposición 4.3 Si $[\cdot, \cdot]$ es el conmutador y $f, g \in S$, entonces

$$
\left\|\left[J^{s}, f\right] g\right\|_{L^{2}} \leq C\left\|\partial_{x} f\right\|_{H^{s-1}}\|g\|_{H^{s-1}},
$$

en donde $s>3 / 2$. 
Demostración : Por el teorema (5.4) tenemos

$$
\left\|\left[J^{s}, f\right] g\right\| \leq C\left(\left\|\widehat{\partial_{x} f}\right\|_{L^{1}}\|g\|_{H^{s-1}}+\left\|\partial_{x} f\right\|_{H^{s-1}}\|\widehat{g}\|_{L^{1}}\right),
$$

dado que $s>3 / 2$. Pero

$$
\begin{aligned}
\left\|\widehat{\partial_{x} f}\right\|_{L^{1}} & =\int_{\mathbb{R}}\left|\widehat{\partial_{x} f}(\xi)\right| d \xi=\int_{\mathbb{R}} \frac{\left(1+\xi^{2}\right)^{\frac{s-1}{2}}}{\left(1+\xi^{2}\right)^{\frac{s-1}{2}}}\left|\widehat{\partial_{x} f}(\xi)\right| d \xi \\
& \leq\left(\int_{\mathbb{R}} \frac{1}{\left(1+\xi^{2}\right)^{s-1}} d \xi\right)^{\frac{1}{2}}\left(\int_{\mathbb{R}}\left(1+\xi^{2}\right)^{s-1}\left|\widehat{\partial_{x} f}(\xi)\right|^{2} d \xi\right)^{\frac{1}{2}} \\
& =C\left\|\partial_{x} f\right\|_{H^{s-1}} .
\end{aligned}
$$

De manera análoga $\|\widehat{g}\|_{L^{1}} \leq C\|g\|_{H^{s-1}}$ y esto demuestra la proposición.

Proposición 4.4 Para todo $u \in S$ se cumple que

$$
\left\|[S, A(t, y)] S^{-1} u\right\|_{L^{2}} \leq s C_{H^{s}}\left\|M_{t} y\right\|_{H^{s}}\|u\|_{L^{2}},
$$

Demostración : Sea $u \in \mathcal{S}$, entonces

$$
\begin{aligned}
\left\|[S, A(t, y)] S^{-1} u\right\|_{L^{2}} & =\left\|e^{t \partial_{x}^{3}}\left[S, M_{t} y\right] \partial_{x} S^{-1} e^{-t \partial_{x}^{3}} u\right\|_{L^{2}} \\
& \leq\left\|e^{t \partial_{x}^{3}}\right\|_{\mathcal{L}\left(L^{2}\right)}\left\|\left[S, M_{t} y\right] S^{-1} \partial_{x} e^{-t \partial_{x}^{3}} u\right\|_{L^{2}} \\
& \leq\left\|\left[S, M_{t} y\right] S^{-1} \partial_{x} e^{-t \partial_{x}^{3}} u\right\|_{L^{2}},
\end{aligned}
$$

y por la proposición anterior tenemos

$$
\begin{aligned}
& \left\|[S, A(t, y)] S^{-1} u\right\|_{L^{2}} \leq C\left\|\partial_{x} M_{t} y\right\|_{H^{s-1}} \| S^{-1} \partial_{x} e^{-t \partial_{x}^{3} u \|_{H^{s-1}}} \\
& \leq C\left\|\partial_{x} M_{t} y\right\|_{H^{s-1}} \| \partial_{x} S^{-1} e^{-t \partial_{x}^{3} u \|_{H^{s-1}}} \\
& \leq C_{s}\left\|M_{t} y\right\|_{H^{s}}\left\|S^{-1} e^{-t \partial_{x}^{3}} u\right\|_{H^{s}} \\
& =C_{s}\left\|M_{t} y\right\|_{H^{s}} \| e^{-t \partial_{x}^{3} u \|_{L^{2}}} \\
& \leq C_{s}\left\|M_{t} y\right\|_{H^{s}}\|u\|_{L^{2}} \text {, }
\end{aligned}
$$

lo que completa la prueba. 
Ahora, para cada $y \in W$, definimos el operador lineal $\widetilde{B}(y)$ por

$$
\left\{\begin{array}{l}
\mathcal{D}(\widetilde{B}(t, y))=\mathcal{S} \\
\widetilde{B}(t, y) u=[S, A(t, y)] S^{-1} u,
\end{array}\right.
$$

Entonces, para todo $u \in \mathcal{S}$ tenemos que

$$
\|\widetilde{B}(t, y) u\|_{L^{2}} \leq C_{s}\left\|M_{t} y\right\|_{H^{s}}\|u\|_{L^{2}} .
$$

Así, $\widetilde{B}(t, y)$ es un operador lineal acotado. Como $\mathcal{S}$ es denso en $X$, extendemos $\widetilde{B}(t, y)$ a $X$ por continuidad y obtenemos el operador lineal $B(t, y) \in \mathcal{L}(X)$ tal que

$$
\|B(t, y)\|_{\mathcal{L}(X)} \leq C_{s}\left\|M_{t} y\right\|_{H^{s}}=\lambda_{B},
$$

para cada $(t, y) \in[0,+\infty[\times W$.

Veamos ahora la siguiente proposición.

Proposición 4.5 Para cada $(t, y) \in[0,+\infty] \times W$ tenemos que $\mathcal{D}\left(S A(t, y) S^{-1}\right)=\mathcal{D}(A(t, y)) y$

$$
S A(t, y) S^{-1}=A(t, y)+B(t, y) .
$$

Demostración : Sea $y \in W, u \in \mathcal{D}(A(t, y))=H^{s} e\left\{u_{n}\right\}_{n \in \mathbf{N}}$ una sucesión en $\mathcal{S}$ tal que $u_{n} \rightarrow$ u en $H^{s}$. Entonces por (17)

$$
A(t, y) S^{-1} u_{n}=S^{-1}\left(A(t, y) u_{n}+\tilde{B}(t, y) u_{n}\right),
$$

y como $B(t, y) u=\lim _{n \rightarrow \infty} \tilde{B}(t, y) u_{n}$ obtenemos que

$$
A(t, y) S^{-1} u_{n} \stackrel{X}{\longrightarrow} S^{-1}(A(t, y) u+B(t, y) u) \text { cuando } n \rightarrow \infty \text {. }
$$

Por otro lado $\lim _{n \rightarrow \infty} S^{-1} u_{n}=S^{-1} u$ en $X$, y como $A(t, y)$ es un operador cerrado tenemos que $S^{-1} u \in \mathcal{D}(A(t, y)) y$

$$
A(t, y) S^{-1} u_{n} \stackrel{X}{\longrightarrow} A(t, y) S^{-1} u \text { cuando } n \rightarrow \infty \text {. }
$$


Luego

$$
A(t, y) S^{-1} u=S^{-1}(A(t, y) u+B(t, y) u) \in H^{s}
$$

Por tanto, $u \in \mathcal{D}\left(S A(t, y) S^{-1}\right) y$

$$
S A(t, y) S^{-1} u=A(t, y) u+B(t, y) u
$$

esto prueba que $S A(t, y) S^{-1}$ es una extensión de $A(t, y)+B(t, y)$. Además, si $\lambda>\omega$ entonces $\lambda \in \rho(A(t, y)) y$

$$
S(A(t, y)-\lambda I) S^{-1} u=A(t, y) u+B(t, y) u-\lambda u \text { para } u \in H^{s} .
$$

Por lo tanto

$$
S(A(t, y)-\lambda I) S^{-1}=A(t, y)+B(t, y)-\lambda I
$$

de donde

$$
S A(t, y) S^{-1}=A(t, y)+B(t, y) .
$$

Lo que prueba la proposición.

Así tenemos verificada la hipótesis (A2).

\subsection{Hipótesis (A3)}

Vamos a probar las siguientes proposiciones.

Proposición 4.6 Para cada $(t, y) \in[0,+\infty] \times W$ tenemos $A(t, y) \in \mathcal{L}(Y, X)$. Demostración : Para cada $(t, y) \in\left[0, T_{0}\right] \times W$ tenemos que $\mathcal{D}(A(t, y)) \supseteq H^{s}=Y$. 
Además, para $u \in Y$ tenemos

$$
\left\|M_{t} y \partial_{x} e^{-t \partial_{x}^{3}} u\right\|_{L^{2}} \leq\left\|M_{t} y\right\|_{L^{\infty}}\|u\|_{H^{s}},
$$

pues

$$
\begin{aligned}
& \left\|M_{t} y \partial_{x} e^{-t \partial_{x}^{3} u}\right\|_{L^{2}}^{2}=\int_{\mathbb{R}}\left|M_{t} y(x) \partial_{x} e^{-t \partial_{x}^{3}} u(x)\right|^{2} d x \\
& \leq\left\|M_{t} y\right\|_{L^{\infty}}^{2} \int_{\mathbb{R}}\left|\partial_{x} e^{-t \partial_{x}^{3}} u\right|^{2} d x \\
& =\left\|M_{t} y\right\|_{L^{\infty}}^{2}\left\|\partial_{x} e^{-t \partial_{x}^{3}} u\right\|_{L^{2}}^{2} \\
& \leq\left\|M_{t} y\right\|_{L^{\infty}}^{2}\left\|\partial_{x} e^{-t \partial_{x}^{3}} u\right\|_{H^{s-1}}^{2}
\end{aligned}
$$

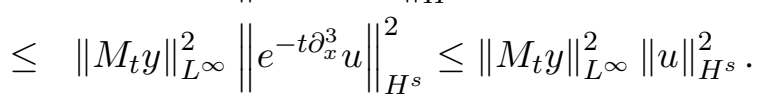

Así,

$$
\begin{aligned}
\|A(t, y) u\|_{L^{2}} & =\left\|e^{t \partial_{x}^{3}} M_{t} y \partial_{x} e^{-t \partial_{x}^{3}} u\right\|_{L^{2}} \\
& \leq\left\|e^{t \partial_{x}^{3}}\right\|_{\mathcal{L}\left(L^{2}\right)}\left\|M_{t} y \partial_{x} e^{-t \partial_{x}^{3}} u\right\|_{L^{2}} \\
& \leq\left\|M_{t} y \partial_{x} e^{-t \partial_{x}^{3}} u\right\|_{L^{2}} \leq\left\|M_{t} y\right\|_{L^{\infty}}\|u\|_{H^{s}} .
\end{aligned}
$$

Por tanto, $A(t, y)$ es un operador lineal acotado de $Y$ en $X$.

Proposición 4.7 Para cada $y \in W$ la aplicación $t \in[0,+\infty[\mapsto A(t, y)$ es fuertemente continua.

Demostración : Sean y $\in W$ y $t_{0} \in[0,+\infty[$ fijos, entonces para $u \in Y$ tenemos

$$
\begin{aligned}
A(t, y) u-A\left(t_{0}, y\right) u= & e^{t \partial_{x}^{3}} M_{t} y \partial_{x} e^{-t \partial_{x}^{3}} u-e^{t_{0} \partial_{x}^{3}} M_{0} y \partial_{x} e^{-t_{0} \partial_{x}^{3}} u \\
= & e^{t \partial_{x}^{3}} M_{t} y \partial_{x} e^{-t \partial_{x}^{3}} u-e^{t \partial_{x}^{3}} M_{0} y \partial_{x} e^{-t_{0} \partial_{x}^{3}} u \\
& +e^{t \partial_{x}^{3}} M_{0} y \partial_{x} e^{-t_{0} \partial_{x}^{3}} u-e^{t_{0} \partial_{x}^{3}} M_{0} y \partial_{x} e^{-t_{0} \partial_{x}^{3} u} \\
= & e^{t \partial_{x}^{3}} M_{t} y e^{-t \partial_{x}^{3}} \partial_{x} u-e^{t \partial_{x}^{3}} M_{0} y e^{-t_{0} \partial_{x}^{3}} \partial_{x} u \\
& +e^{t \partial_{x}^{3}} M_{0} y \partial_{x} e^{-t_{0} \partial_{x}^{3}} u-e^{t_{0} \partial_{x}^{3}} M_{0} y \partial_{x} e^{-t_{0} \partial_{x}^{3}} u .
\end{aligned}
$$




\section{Entonces}

$$
\begin{aligned}
& \left\|A(t, y) u-A\left(t_{0}, y\right) u\right\|_{L^{2}} \leq\left\|e^{t \partial_{x}^{3}} M_{t} y e^{-t \partial_{x}^{3}} \partial_{x} u-e^{t \partial_{x}^{3}} M_{0} y e^{-t_{0} \partial_{x}^{3}} \partial_{x} u\right\|_{L^{2}} \\
& +\left\|e^{t \partial_{x}^{3}} M_{0} y \partial_{x} e^{-t_{0} \partial_{x}^{3}} u-e^{t_{0} \partial_{x}^{3}} M_{0} y \partial_{x} e^{-t_{0} \partial_{x}^{3}} u\right\|_{L^{2}} \\
& =\left\|e^{t \partial_{x}^{3}}\left(M_{t} y e^{-t \partial_{x}^{3}} \partial_{x} u-M_{0} y e^{-t_{0} \partial_{x}^{3}} \partial_{x} u\right)\right\|_{L^{2}} \\
& +\left\|\left(e^{t \partial_{x}^{3}}-e^{t_{0} \partial_{x}^{3}}\right) M_{0} y \partial_{x} e^{-t_{0} \partial_{x}^{3}} u\right\|_{L^{2}} \\
& \leq\left\|e^{t \partial_{x}^{3}}\right\|_{\mathcal{L}\left(L^{2}\right)}\left\|M_{t} y e^{-t \partial_{x}^{3}} \partial_{x} u-M_{0} y e^{-t_{0} \partial_{x}^{3}} \partial_{x} u\right\|_{L^{2}} \\
& +\left\|e^{t \partial_{x}^{3}}-e^{t_{0} \partial_{x}^{3}}\right\|_{\mathcal{L}\left(L^{2}\right)}\left\|M_{0} y \partial_{x} e^{-t_{0} \partial_{x}^{3}} u\right\|_{L^{2}} \\
& \leq\left\|M_{t} y e^{-t \partial_{x}^{3}} \partial_{x} u-M_{0} y e^{-t_{0} \partial_{x}^{3}} \partial_{x} u\right\|_{L^{2}} \\
& +\left\|e^{t \partial_{x}^{3}}-e^{t_{0} \partial_{x}^{3}}\right\|_{\mathcal{L}\left(L^{2}\right)}\left\|M_{0} y \partial_{x} e^{-t_{0} \partial_{x}^{3}} u\right\|_{L^{2}} .
\end{aligned}
$$

Pero

$$
\begin{aligned}
\left\|M_{t} y e^{-t \partial_{x}^{3}} \partial_{x} u-M_{0} y e^{-t_{0} \partial_{x}^{3}} \partial_{x} u\right\|_{L^{2}} \leq & \left\|M_{t} y e^{-t \partial_{x}^{3}} \partial_{x} u-M_{0} y e^{-t_{0} \partial_{x}^{3}} \partial_{x} u\right\|_{L^{2}} \\
& +\left\|M_{t} y e^{-t_{0} \partial_{x}^{3}} \partial_{x} u-M_{0} y e^{-t_{0} \partial_{x}^{3}} \partial_{x} u\right\|_{L^{2}} \\
\leq & \left\|M_{t} y\right\|_{L^{\infty}}\left\|\left[e^{-t \partial_{x}^{3}}-e^{-t_{0} \partial_{x}^{3}}\right] \partial_{x} u\right\|_{s} \\
& +\left\|\left[M_{t} y-M_{0} y\right] e^{-t_{0} \partial_{x}^{3}} \partial_{x} u\right\|_{L^{2}} \\
\leq & \left\|M_{t} y\right\|_{L^{\infty}}\left\|e^{t \partial_{x}^{3}}-e^{t_{0} \partial_{x}^{3}}\right\|_{\mathcal{L}\left(L^{2}\right)}\left\|\partial_{x} u\right\|_{H^{s-1}} \\
& +\left\|\left[M_{t} y-M_{0} y\right] e^{-t_{0} \partial_{x}^{3}} \partial_{x} u\right\|_{L^{2}} .
\end{aligned}
$$

Sustituyendo en (18) resulta

$$
\begin{aligned}
\left\|A(t, y) u-A\left(t_{0}, y\right) u\right\|_{L^{2}} \leq & \left\|M_{t} y\right\|_{L^{\infty}}\left\|e^{t \partial_{x}^{3}}-e^{t_{0} \partial_{x}^{3}}\right\|_{\mathcal{L}\left(L^{2}\right)}\left\|\partial_{x} u\right\|_{H^{s-1}} \\
& +\left\|\left[M_{t} y-M_{0} y\right] e^{-t_{0} \partial_{x}^{3}} \partial_{x} u\right\|_{L^{2}} \\
& +\left\|e^{t \partial_{x}^{3}}-e^{t_{0} \partial_{x}^{3}}\right\|_{\mathcal{L}\left(L^{2}\right)}\left\|M_{0} y \partial_{x} e^{-t_{0} \partial_{x}^{3}} u\right\|_{L^{2}} .
\end{aligned}
$$


Notemos que $M_{t} y$ es fuertemente continua,

$$
\begin{aligned}
\left\|M_{t} y-M_{0} y\right\|_{H^{s}} & =\left\|\left(\sum_{j=1}^{p}\left(M_{t} y\right)^{\frac{p-j}{p}}\left(N_{y}^{0}\right)^{\frac{j-1}{p}}\right)\left(\left(M_{t} y\right)^{\frac{1}{p}}-\left(M_{0} y\right)^{\frac{1}{p}}\right) y\right\|_{H^{s}} \\
& \leq\left\|\left(e^{-t \partial_{x}^{3}}-e^{-t_{0} \partial_{x}^{3}}\right) y\right\|_{H^{s}} \sum_{j=1}^{p}\left\|M_{0} y\right\|_{H^{s}}^{\frac{p-j}{p}}\left\|N_{y}^{0}\right\|_{H^{s}}^{\frac{j-1}{p}} \\
& \leq\left\|e^{t \partial_{x}^{3}}-e^{t_{0} \partial_{x}^{3}}\right\|_{\mathcal{L}\left(L^{2}\right)}\|y\|_{H^{s}} \sum_{j=1}^{p}\left\|M_{t} y\right\|_{H^{s}}^{\frac{p-j}{p}}\left\|M_{0} y\right\|_{H^{s}}^{\frac{j-1}{p}} \\
& \leq p\left\|e^{t \partial_{x}^{3}}-e^{t_{0} \partial_{x}^{3}}\right\|_{\mathcal{L}\left(L^{2}\right)}\|y\|_{H^{s}}^{p-1},
\end{aligned}
$$

por lo que

$$
\left\|M_{t} y-M_{0} y\right\|_{H^{s}} \leq p\left\|e^{t \partial_{x}^{3}}-e^{t_{0} \partial_{x}^{3}}\right\|_{\mathcal{L}\left(L^{2}\right)}\|y\|_{H^{s}}^{p-1} \rightarrow 0 \text { cuando } t \rightarrow t_{0} .
$$

Así tenemos

$$
\begin{array}{r}
\left\|M_{t} y\right\|_{L^{\infty}}\left\|e^{t \partial_{x}^{3}}-e^{t_{0} \partial_{x}^{3}}\right\|_{\mathcal{L}\left(L^{2}\right)}\left\|\partial_{x} u\right\|_{H^{s-1}} \\
\left\|\left(M_{t} y-M_{0} y\right) e^{-t_{0} \partial_{x}^{3}} \partial_{x} u\right\|_{L^{2}} \rightarrow 0 \text { cuando } t \rightarrow t_{0},
\end{array}
$$

$y$

$$
\left\|e^{t \partial_{x}^{3}}-e^{t_{0} \partial_{x}^{3}}\right\|_{\mathcal{L}\left(L^{2}\right)}\left\|M_{0} y \partial_{x} e^{-t_{0} \partial_{x}^{3}} u\right\|_{L^{2}} \rightarrow 0 \text { cuando } t \rightarrow t_{0}
$$

por lo tanto, en (19).

Así hemos verificada la hipótesis (A3).

\subsection{Hipótesis (A4)}

Tenemos la siguiente proposición. 
Proposición 4.8 Para cada $t \in\left[0,+\infty\left[\right.\right.$ existe $\mu_{A}>0$ tal que

$$
\left\|A\left(t, y_{1}\right)-A\left(t, y_{2}\right)\right\|_{\mathcal{L}(X)} \leq \mu_{A}\left\|y_{1}-y_{2}\right\|_{Y},
$$

para todo $y_{1}, y_{2} \in W$.

Demostración : Si $y_{1}, y_{2} \in W$ entonces para $u \in Y$

$$
\begin{aligned}
A\left(t, y_{1}\right) u-A\left(t, y_{2}\right) u & =e^{t \partial_{x}^{3}} M_{t} y_{1} \partial_{x} e^{-t \partial_{x}^{3}} u-e^{t \partial_{x}^{3}} M_{t} y_{2} \partial_{x} e^{-t \partial_{x}^{3}} u \\
& =e^{t \partial_{x}^{3}}\left(M_{t} y_{1}-M_{t} y_{2}\right) \partial_{x} e^{-t \partial_{x}^{3}} u
\end{aligned}
$$

así,

$$
\begin{aligned}
& \left\|A\left(t, y_{1}\right) u-A\left(t, y_{2}\right) u\right\|_{L^{2}}=\left\|e^{t \partial_{x}^{3}}\left(M_{t} y_{1}-M_{t} y_{2}\right) \partial_{x} e^{-t \partial_{x}^{3}} u\right\|_{L^{2}} \\
& \leq\left\|\left(M_{t} y_{1}-M_{t} y_{2}\right) \partial_{x} e^{-t \partial_{x}^{3}} u\right\|_{H^{s-1}} \\
& \leq\left\|M_{t} y_{1}-M_{t} y_{2}\right\|_{H^{s-1}}\left\|\partial_{x} e^{-t \partial_{x}^{3}} u\right\|_{H^{s-1}} \\
& \leq\left\|M_{t} y_{1}-M_{t} y_{2}\right\|_{H^{s}}\left\|e^{-t \partial_{x}^{3} u}\right\|_{H^{s}} \\
& \leq \sum_{j=1}^{p}\left\|\left(M_{t} y_{1}\right)^{\frac{p-j}{p}}\left(M_{t} y_{2}\right)^{\frac{j-1}{p}}\left(y_{1}-y_{2}\right)\right\|_{H^{s}}\|u\|_{H^{s}} \text {. }
\end{aligned}
$$

usando (21) resulta

$$
\begin{aligned}
\left\|A\left(t, y_{1}\right) u-A\left(t, y_{2}\right) u\right\|_{L^{2}} & \leq \sum_{j=1}^{p} R^{p-1}\left\|y_{1}-y_{2}\right\|_{H^{s}}\|u\|_{H^{s}} \\
& =p R^{p-1}\left\|y_{1}-y_{2}\right\|_{H^{s}}\|u\|_{H^{s}} .
\end{aligned}
$$

Por tanto

$$
\left\|A\left(t, y_{1}\right)-A\left(t, y_{2}\right)\right\|_{\mathcal{L}\left(L^{2}\right)} \leq p R^{p-1}\left\|y_{1}-y_{2}\right\|_{H^{s}},
$$

y la prueba está completa.

Así hemos verificada la hipótesis (A4). 


\subsection{Hipótesis (A5)}

Proposición 4.9 Existe $\mu_{B}>0$ tal que

$$
\left\|B\left(t, y_{1}\right)-B\left(t, y_{2}\right)\right\|_{\mathcal{L}(X)} \leq \mu_{B}\left\|y_{1}-y_{2}\right\|_{H^{s}},
$$

para cada $\left(t, y_{1}\right),\left(t, y_{2}\right) \in[0,+\infty[\times W$.

Demostración : De la proposición 4.7 tenemos

$$
\begin{aligned}
B(t, y) & =S e^{t \partial_{x}^{3}} M_{t} y \partial_{x} e^{-t \partial_{x}^{3}} S^{-1}-e^{t \partial_{x}^{3}} M_{t} y \partial_{x} e^{-t \partial_{x}^{3}} \\
& =S e^{t \partial_{x}^{3}} M_{t} y \partial_{x} e^{-t \partial_{x}^{3}} S^{-1}-e^{t \partial_{x}^{3}} M_{t} y S \partial_{x} e^{-t \partial_{x}^{3}} S^{-1} \\
& =\left(S e^{t \partial_{x}^{3}} M_{t} y-e^{t \partial_{x}^{3}} M_{t} y S\right) \partial_{x} e^{-t \partial_{x}^{3}} S^{-1},
\end{aligned}
$$

luego

$$
\begin{aligned}
& B\left(t, y_{1}\right)-B\left(t, y_{2}\right) \\
& =\left(S e^{t \partial_{x}^{3}} M_{t} y_{1}-e^{t \partial_{x}^{3}} M_{t} y_{1} S\right) \partial_{x} e^{-t \partial_{x}^{3}} S^{-1}-\left(S e^{t \partial_{x}^{3}} M_{t} y_{2}-e^{t \partial_{x}^{3}} M_{t} y_{2} S\right) \partial_{x} e^{-t \partial_{x}^{3}} S^{-1} \\
& =\left(S e^{t \partial_{x}^{3}}\left(M_{t} y_{1}-M_{t} y_{2}\right)-e^{t \partial_{x}^{3}}\left(M_{t} y_{1}-M_{t} y_{2}\right) S\right) \partial_{x} e^{-t \partial_{x}^{3}} S^{-1} \\
& =\left(S, e^{t \partial_{x}^{3}}\left(M_{t} y_{1}-M_{t} y_{2}\right)\right) \partial_{x} e^{-t \partial_{x}^{3}} S^{-1}
\end{aligned}
$$

\section{Como}

$$
M_{t} y_{1}-M_{t} y_{2}=\left(\sum_{j=1}^{p}\left(M_{t} y_{1}\right)^{\frac{p-j}{p}}\left(M_{t} y_{2}\right)^{\frac{j-1}{p}}\right) e^{-t \partial_{x}^{3}}\left(y_{1}-y_{2}\right)
$$

entonces

$$
\begin{aligned}
{\left[S, e^{t \partial_{x}^{3}}\left(M_{t} y_{1}-M_{t} y_{2}\right)\right] } & =\left[S,\left(\sum_{j=1}^{p}\left(M_{t} y_{1}\right)^{\frac{p-j}{p}}\left(M_{t} y_{2}\right)^{\frac{j-1}{p}}\right) e^{-t \partial_{x}^{3}}\left(y_{1}-y_{2}\right)\right] \\
& =\sum_{j=1}^{p}\left[S,\left(M_{t} y_{1}\right)^{\frac{p-j}{p}}\left(M_{t} y_{2}\right)^{\frac{j-1}{p}} e^{-t \partial_{x}^{3}}\left(y_{1}-y_{2}\right)\right],
\end{aligned}
$$

luego

$$
B\left(t, y_{1}\right)-B\left(t, y_{2}\right)=\sum_{j=1}^{p}\left[S,\left(M_{t} y_{1}\right)^{\frac{p-j}{p}}\left(M_{t} y_{2}\right)^{\frac{j-1}{p}} e^{-t \partial_{x}^{3}}\left(y_{1}-y_{2}\right)\right] \partial_{x} e^{-t \partial_{x}^{3}} S^{-1}
$$




\section{Por tanto}

$$
\begin{aligned}
& \left\|B\left(t, y_{1}\right) u-B\left(t, y_{2}\right) u\right\|_{L^{2}} \\
& =\left\|\sum_{j=1}^{p}\left[S, e^{t \partial_{x}^{3}}\left(M_{t} y_{1}\right)^{\frac{p-j}{p}}\left(M_{t} y_{2}\right)^{\frac{j-1}{p}} e^{-t \partial_{x}^{3}}\left(y_{1}-y_{2}\right)\right]\right\|_{L^{2}} \\
& \leq \sum_{j=1}^{p}\left\|\left[S, e^{t \partial_{x}^{3}}\left(M_{t} y_{1}\right)^{\frac{p-j}{p}}\left(M_{t} y_{2}\right)^{\frac{j-1}{p}} e^{-t \partial_{x}^{3}}\left(y_{1}-y_{2}\right)\right] \partial_{x} e^{-t \partial_{x}^{3}} S^{-1}\right\|_{L^{2}} \\
& \leq C \sum_{j=1}^{p}\left\|\partial_{x} e^{t \partial_{x}^{3}}\left(M_{t} y_{1}\right)^{\frac{p-j}{p}}\left(M_{t} y_{2}\right)^{\frac{j-1}{p}} e^{-t \partial_{x}^{3}}\left(y_{1}-y_{2}\right)\right\|_{H^{s-1}}\left\|\partial_{x} e^{-t \partial_{x}^{3}} S^{-1} u\right\|_{H^{s-1}} \\
& \leq C_{s} \sum_{j=1}^{p}\left\|e^{t \partial_{x}^{3}}\left(M_{t} y_{1}\right)^{\frac{p-j}{p}}\left(M_{t} y_{2}\right)^{\frac{j-1}{p}} e^{-t \partial_{x}^{3}}\left(y_{1}-y_{2}\right)\right\|_{H^{s}}\|u\|_{L^{2}},
\end{aligned}
$$

pero

$$
\begin{aligned}
& \left\|e^{t \partial_{x}^{3}}\left(M_{t} y_{1}\right)^{\frac{p-j}{p}}\left(M_{t} y_{2}\right)^{\frac{j-1}{p}} e^{-t \partial_{x}^{3}}\left(y_{1}-y_{2}\right)\right\|_{H^{s}} \\
\leq & \left\|\left(M_{t} y_{1}\right)^{\frac{p-j}{p}}\left(M_{t} y_{2}\right)^{\frac{j-1}{p}}\right\|_{H^{s}}\left\|y_{1}-y_{2}\right\|_{H^{s}} \\
\leq & \left\|y_{1}\right\|_{H^{s}}^{p-j}\left\|y_{2}\right\|_{H^{s}}^{j-1}\left\|y_{1}-y_{2}\right\|_{H^{s}} \\
\leq & R^{p-1}\left\|y_{1}-y_{2}\right\|_{H^{s}} .
\end{aligned}
$$

Por tanto, en (20) resulta

$$
\left\|B\left(t, y_{1}\right) u-B\left(t, y_{2}\right) u\right\|_{L^{2}} \leq C_{s} p R^{p-1}\left\|y_{1}-y_{2}\right\|_{H^{s}}\|u\|_{L^{2}} .
$$

Esto completa la prueba.

Así hemos verificado la hipótesis (A5).

\subsection{Hipótesis (f1) y (f2)}

Como en nuestro caso, $f(t, u(t))=0$, las hipótesis (f1) y (f2) son trivialmente satisfechas.

En consecuencia, del teorema (5), tenemos el resultado principal de este trabajo. 


\section{Teorema 4.1}

1. Sea $s>3 / 2$. Para cada $u_{0} \in H^{s}$, existe $T>0$, una solución única $u$ para $(K d V g)$ tal que

$$
u \in C\left([0, T]: H^{s}\right) \cap C^{1}\left([0, T]: H^{s-3}\right) .
$$

2. La aplicación $u_{0} \in H^{s} \mapsto u \in C\left([0, T], H^{s}\right)$ es continua en la norma de $H^{s}$. Con mayor precisión, si $u_{n} \in H^{s}, n \in \mathbb{N}$, con $\left\|u_{n}-u\right\|_{H^{s}} \rightarrow 0$ y $T^{\prime}<T$, la solución $u_{n}$ para $u_{n}(0)=u_{0, n}$ existe en $\left[0, T^{\prime}\right]$ para un $n$ suficientemente grande $y\left\|u_{n}(t)-u(t)\right\|_{H^{s}} \rightarrow 0$ uniformemente en $t \in\left[0, T^{\prime}\right]$.

\section{Teoremas adicionales}

Teorema 5.1 Si $u \in L^{1}\left(\mathbb{R}^{n}\right) \cap L^{2}\left(\mathbb{R}^{n}\right)$, entonces $\widehat{u} \in L^{2}\left(\mathbb{R}^{n}\right) y$

$$
\|\widehat{u}\|_{L^{2}}=\|u\|_{L^{2}} .
$$

Demostración : Ver [12].

El teorema de Plancherel afirma que

$$
\widehat{\cdot}: L^{1}\left(\mathbb{R}^{n}\right) \cap L^{2}\left(\mathbb{R}^{n}\right) \rightarrow L^{2}\left(\mathbb{R}^{n}\right)
$$

definido sobre el subespacio denso $L^{1}\left(\mathbb{R}^{n}\right) \cap L^{2}\left(\mathbb{R}^{n}\right)$ de $L^{2}\left(\mathbb{R}^{n}\right)$ es un operador lineal acotado. Por esto existe una extensión única

$$
\mathcal{F}: L^{2}\left(\mathbb{R}^{n}\right) \rightarrow L^{2}\left(\mathbb{R}^{n}\right),
$$

del operador definido en (23). 
Teorema 5.2 Para todo $\alpha \in \mathbb{N}^{n}$ y para todo $s \in \mathbb{R}$,

$$
D^{\alpha}: H^{s}\left(\mathbb{R}^{n}\right) \rightarrow H^{s-|\alpha|}\left(\mathbb{R}^{n}\right)
$$

es un operador lineal acotado, $y$

$$
\left\|D^{\alpha} u\right\|_{H^{s-|\alpha|}} \leq\|u\|_{H^{s}} .
$$

Demostración : Ver [4].

En el siguiente teorema, $C_{\infty}^{k}\left(\mathbb{R}^{n}\right)$ el espacio de las funciones $u \in C^{k}$ tales que $\lim _{|x| \rightarrow \infty} \partial^{\alpha} u(x)=0$ para todo multi-índice $\alpha$ con $|\alpha| \leq k$, es provisto de la norma

$$
\|u\|_{C_{\infty}^{k}}=\max _{|\alpha| \leq k}\left\|\partial^{\alpha} u\right\|_{L^{\infty}}
$$

Teorema 5.3 Sean $s \in \mathbb{R}, k, n \in \mathbb{N}$ con $s>\frac{n}{2}+k$, entonces $H^{s}\left(\mathbb{R}^{n}\right) \hookrightarrow C_{\infty}^{k}\left(\mathbb{R}^{n}\right)$. Además, en tal caso, si $u \in H^{s}\left(\mathbb{R}^{n}\right)$ tenemos

$$
\left\|\partial^{\alpha} u\right\|_{L^{\infty}} \leq \max _{|\alpha| \leq k}\left\|\partial^{\alpha} u\right\|_{L^{\infty}} \leq C_{s}\|u\|_{H^{s}}
$$

para todo multi-índice $\alpha$ con $|\alpha| \leq k$.

Demostración : Ver [12].

Teorema 5.4 Si $[\cdot, \cdot]$ es el conmutador y $f, g \in \mathcal{S}$, entonces

$$
\left\|\left[J^{s}, f\right] g\right\| \leq C\left(\left\|\widehat{\partial_{x} f}\right\|_{L^{1}}\|g\|_{H^{s-1}}+\left\|\partial_{x} f\right\|_{H^{s-1}}\|\widehat{g}\|_{L^{1}}\right)
$$

con $s \geq 1$.

Demostración : Ver [7]. 


\section{Conclusiones}

Al tratar de resolver (4) basándonos en el método de aproximaciones denominado el teorema de punto fijo de Banach, vemos que no es posible obtener una solución, debido al operador $\left\{e^{-t \partial_{x}^{3}}\right\}$ aplica $H^{s-1}$ en $H^{s-1}$ y no en $H^{s}$. La teoría de Kato, permite resolver (4) por el método tradicional, pero en la hipótesis (A3) se considera al operador $A(t, y)$ como fuertemente continua a diferencia de la hipótesis original, en donde $A(t, y)$ es continua, así la solución local en el espacio $H^{s}$ con $s>3 / 2$ se verifica.

\section{Referencias}

[1] Arbogast, T.; Bona, J. (2005) Method of Applied Mathematics. Department of Mathematics the University of Texas at Austin Fall and Spring Semesters, USA.

[2] Bona, J; Smith, R. (1975) "The initial-value problem for the Korteweg-de Vries equation", Philosophical Transactions of the Royal Society of London. Series A, Mathematical and Physical Sciences 278(1287): 555-601.

[3] Darmois G.(1974) Evolution Equation in a Banach Space. Doctorate Thesis, Departament of Mathematics, University of California, California.

[4] Iório, R.J.; Iório, V. (1988) Equações Diferenciais Parciais: Uma Introdução. Projeto Euclides, IMPA. Rio de Janeiro.

[5] Iório, R.J.; Iório, V. (2001) Fourier Analysis and Partial Differential Equations. Cambridge University Press, New York.

[6] Kato, T. (1975) "Quasi linear equations of evolution with application to partial differential equations", Spectral Theory and Differential Equations 448: $25-70$. 
[7] Kato, T.(1983) "On the Cauchy problem for the (Generalized) KdV equations", Studies in Applied Mathematics, Advances in Mathematics Supplementary Studies 8: 93-128.

[8] Kobayashi, K. (1979) "On a theorem for linear evolution equations of hyperbolic type", Journal of the Mathematical Society of Japan 31(4): 647654.

[9] Montealegre, J.; Petrozzi, S. (1998) Operadores disipativos maximales. Informe de Investigación, $\mathrm{N}^{\circ} 2$ Serie B, Pontificia Universidad Católica del Perú, Lima.

[10] Montealegre, J.; Petrozzi, S. (1999) Semigrupos de operadores lineales y ecuaciones de evolución semi-lineales. Informe de Investigación, $\mathrm{N}^{\circ} 6$ Serie B, Pontificia Universidad Católica del Perú, Lima.

[11] Saut, J.C.; Temam, R. (1976) "Remarks on the Korteweg-de Vries equation", Israel Journal of Mathematics 24(1): 78-87.

[12] Zimmer, R.J. (1990) Essential Results of Functional Analysis. University of Chicago Press, USA. 
